\title{
Flavonoids and cognitive function: a review of human randomized controlled trial studies and recommendations for future studies
}

\author{
Anna L. Macready · Orla B. Kennedy · Judi A. Ellis • \\ Claire M. Williams · Jeremy P. E. Spencer • \\ Laurie T. Butler
}

Received: 19 July 2009/ Accepted: 21 July 2009/Published online: 13 August 2009

(C) Springer-Verlag 2009

\begin{abstract}
Evidence in support of the neuroprotective effects of flavonoids has increased significantly in recent years, although to date much of this evidence has emerged from animal rather than human studies. Nonetheless, with a view to making recommendations for future good practice, we review 15 existing human dietary intervention studies that have examined the effects of particular types of flavonoid on cognitive performance. The studies employed a total of 55 different cognitive tests covering a broad range of cognitive domains. Most studies incorporated at least one measure of executive function/working memory, with nine reporting significant improvements in performance as a function of flavonoid supplementation compared to a control group. However, some domains were overlooked completely (e.g. implicit memory, prospective memory), and for the most part there was little consistency in terms of the particular cognitive tests used making across study comparisons difficult. Furthermore, there was some confusion concerning what aspects of cognitive function particular tests were actually measuring. Overall, while initial results are encouraging, future studies need to pay careful attention when selecting cognitive measures, especially in terms of ensuring that tasks are actually sensitive enough to detect treatment effects.
\end{abstract}

A. L. Macready · J. A. Ellis · C. M. Williams · L. T. Butler ( $\square)$ School of Psychology and Clinical Language Sciences, University of Reading, Earley Gate, Whiteknights, PO Box 238, Reading RG6 6AL, UK

e-mail: 1.t.butler@reading.ac.uk

A. L. Macready - O. B. Kennedy - J. P. E. Spencer

Department of Food and Nutritional Sciences,

School of Chemistry, Food and Pharmacy,

University of Reading, Reading, UK
Keywords Flavonoid - Cognition - Cognitive function · Memory $\cdot$ Executive function $\cdot$ Motor function

\section{Introduction}

With the number of people over the age of 60 expected to double between 2000 and 2050 [71], the projected incidence of age-related neurodegenerative diseases, and related health care costs, is also set to rise significantly. A recent report to the Alzheimer's Society estimated the current annual cost of dementia in the UK alone to be around $£ 17.03$ billion [30]. Research into age-related cognitive decline has found that normal ageing and neurodegenerative diseases such as Alzheimer's disease (AD), vascular dementia (VAD) [25] and Parkinson's disease (PD) [24] can be moderated or mediated by oxidative stress and inflammation. In PD, for instance, the selective and progressive loss of dopaminergic neurons in the substantia nigra may be indirectly caused by oxidation of dopamine in the presence of cellular thiols such as cysteine $[64,70]$. In normal ageing and in cardiovascular diseases, such as atherosclerosis [43], inflammatory processes are understood to be both directly and indirectly responsible for cognitive decline. Investigation of the neuroprotective and cognitive benefits of foods and food components, such as flavonoids which are rich in compounds that may reduce these neurodegenerative and inflammatory effects, is, therefore, an important step in the search for dietary therapies to help maintain cognitive health with age [6,39].

The protective properties of flavonoids show enormous potential in counteracting many aspects of cognitive decline [68] and to date, over 6,000 types of flavonoid have been identified [19]. Flavonoids are made up of two aromatic carbon rings and a benzene ring, and their structure 
varies according to their degree of flavan ring oxidation, their glycosylation or alkylation, and the hydroxylation pattern of their molecules (see Vauzour et al. [68] for information regarding the structure of flavonoids). Flavonoids are divided into six classes: flavonols, flavones, isoflavones, flavanones, flavanols and anthocyanidins [38]. Flavonoids are found in high quantities in vegetables, fruits and fruit juices, and other beverages such as tea, red wine and chocolate; plant extracts from pine bark, Gingko biloba and Pueraria lobata which are high in flavonoids, are regularly consumed for their reputed health-giving properties, in particular, to protect against cognitive decline [35, 41, 48, 53, 57].

Flavonoids are thought to enhance cognitive function via their neuroprotective properties, enhancing neuronal function and by stimulating neurogenesis [61, 62]. They have the capacity to cross the blood-brain barrier, and have been detected in the rat brain in areas related to learning and memory shortly after oral administration [1, 45]. Flavonoids may therefore help to protect against the memory deficits associated with normal ageing as well as the neurodegenerative processes associated with various forms of dementia, such as AD, VAD and PD [24, 34, 59, $63,64]$. A range of possible mechanisms through which flavonoids might reduce cognitive decline have been identified, in particular, their ability to interact with the neuronal intracellular signalling pathways mediating neurodegeneration and neuroinflammation [61, 62].

Flavonoids are thought to work by modulating cellular signalling pathways, such as the mitogen-activated proteinkinase pathway (MAPK) and the phosphoinositide 3-kinase (PI3 kinase/Akt) signalling cascade [73]. They are also known to modulate the transcription factors nuclear factorkappa B (NF-kB), which is involved in signal transduction through protein-kinase inhibition [18], and to promote the expression of brain derived neurotrophic factor (BDNF) [72], which is responsible for neuronal and synaptic growth and survival $[29,72]$. One class of flavonoids, isoflavones, appears to act by mimicking oestrogen, and affecting brain function by mediating oestrogen receptor processes and inhibiting tyrosine kinase [36]. Of particular interest is the ability of flavonoids to improve endothelial function by increasing production of the signalling molecule nitric oxide (NO), leading to relaxation of the endothelial smooth muscle, which in turn results in a greater control of blood pressure [58]. Increased NO inhibits the enzymes nitric oxide synthase (iNOS) and cyclo-oxygenase (COX-2), reactive $\mathrm{C}$ protein and the atheromatous plaque adhesion molecules known to be involved in inflammation [17]. Vascular dysfunction, resulting from endothelial dysfunction, is thought to play a pivotal role in the development and progression of cardiovascular diseases implicated in cognitive decline, such as atherosclerosis, and is therefore also an important mechanism to consider in the development of neurodegenerative disorders, such as AD and PD [5, 15, 20, 52, 67]. Flavonoids have also been shown to reduce oxidative stress via their ability to scavenge reactive oxygen species (ROS), although their in vitro redox potential is greatly reduced in vivo $[62,68]$.

Studies using animal models (reviewed in this volume, see Rendeiro et al. 2009) have demonstrated that certain groups of flavonoids may slow and even reverse the effects of ageing and dementia [16, 27, 28]. In existing rat studies, memory deficits may be prevented by the consumption of foods rich in anthocyanins, a flavonoid subgroup $[3,8,34$, $51,60]$. For example, supplementation in rats has been shown to enhance both long-term memory [3, 8, 34], and spatial working memory [72], resulting in higher levels of brain-derived neurotrophic factor (BDNF) in the hippocampus $[3,8,34,51,60]$. Flavonoid compounds, such as anthocyanins, have been shown to be present in the brain 10 min after consumption and their presence is correlated with improved performance on cognitive tasks relating to learning and memory [1]. One recent rat study showed that blueberry supplementation prevented elevated levels of nuclear factor-kappa B (NF- $\kappa \mathrm{B})$, an oxidative stressresponsive protein, in areas of the brain associated with cognition (frontal and hippocampal) and motor performance (striatum and cerebellum). Memory scores were associated with NF- $\kappa \mathrm{B}$ levels in the hippocampus and cerebellum, suggesting that the cerebellum may play a role in cognitive as well as motor function [18]. Another study found a dramatically attenuated memory impairment correlated with higher levels of BDNF in mice after supplementation by the flavonoid Oroxylin A [29].

While existing animal studies provide crucial insights regarding the neuroprotective properties of flavonoids and their underlying mechanisms, such studies are mute with respect to possible effects on more complex human cognitive functions that involve abilities underpinned by language, such as verbal memory, implicit memory and learning, and those supported by our ability to organise information, such as executive function. Executive function is a complex and somewhat controversial term used to describe a number of distinct, specifiable 'control' functions that are distinguishable from processing speed, memory and motor functions. Examples of executive functions include 'switching' or 'shifting' (e.g. alternating between behaviours or information sources), 'inhibition' (the ability to suppress automatic and habitual responses or behaviours, 'updating' (the ability to discard and replace information [42, 49]), 'sustained attention' (requiring sustained concentration and monitoring skills [40, 49]), 'strategic memory search' (conscious, controlled retrieval of structured information [4, 40, 47, 49]) and 'planning' (the ability to deal with novel information, generate goals 
and make decisions on a suitable course of action [4, 49]). All of the above processes are dependent on 'working memory', a psychological construct used to describe a hypothetical system for the temporary manipulation and maintenance of speech-based and/or visuospatial information, requiring the control of attentional resources [2]. In terms of memory and learning, various distinctions can be drawn between short-term memory (e.g. digit span or digit recall tasks) and long-term memory (e.g. episodic immediate or delayed recall and recognition), conscious or unconscious processes (e.g. explicit versus implicit forms of memory and learning), memory for events (episodic memory) or meaning (semantic memory), remembering to perform actions (prospective memory), memory for skills (procedural memory) and memory for images and/or spatial orientation (visuospatial or spatial memory). Motor function is measured with or without a cognitive component (e.g. motor speed or psychomotor speed), and IQ may be sub-divided into crystallised intelligence (measuring acquired knowledge) and fluid intelligence (measuring non-verbal ability, problem-solving and pattern recognition independent of acquired knowledge). Thus attempts to characterise the effects of flavonoids on human cognition need to utilise a wide range of tasks to fully assess cognitive ability. In doing so, it is important to bear in mind two points. First, although a particular task might be identified as having a primary neuropsychological focus such as 'executive function' or 'episodic memory', such measures are not 'task pure' [4]. For example, a range of processes may support a nominally 'executive', task such as memory, processing speed and motor function. Second, in terms of the underlying brain regions supporting cognitive performance, it is important to recognise that any task is likely to recruit multiple neural regions. For example, functional neuroimaging studies have revealed activations in prefrontal cortex, medial and lateral parietal cortex, as well as hippocampal/medial temporal lobe activations during episodic memory retrieval [56].

The aim of the current paper is to review the methodological approaches used to examine the effects of flavonoid supplementation on cognitive performance in humans. While human epidemiological studies and evidence from animal models suggests a positive association/relationship between flavonoid intake and cognitive function [35, 50], comparably few studies have been conducted using randomised controlled trial (RCT) designs. Nonetheless, with a view to making recommendations for future good practice, it is timely to review the design and methodological considerations of those existing intervention studies. We sought to address four main questions: (1) what proportion of flavonoid interventions have reported significant benefits to cognition and in which domains? (2) How much consistency is there across studies in terms of cognitive domains measured and tasks employed? (3) Are there any cognitive domains that are underrepresented in existing intervention studies? (4) What are the implications for future flavonoid intervention studies?

\section{Methods}

A search of three databases (Web of Knowledge, Medline and the Cochrane Central register of Controlled Trials) for human flavonoid randomised controlled trials exploring cognitive function as the primary or secondary outcome was carried out using the search terms 'Flavonoid + Cognitive' and 'Flavonoid + Memory' for the past 10 years (e.g. January 1999-March 2009). Non-randomised studies and studies that did not include a control and/or placebo group were excluded. Studies that specifically focused on the cognitive effects of flavonoids either in extract or whole food form were included. Outcomes were changes in cognitive performance. Tasks were initially categorised by their primary neuropsychological focus, which was determined by the descriptions provided in the selected review papers. Where disagreement occurred, the authors used the task descriptions most commonly used in the psychology literature for each task. Next, any additional processes measured by each task were identified based on terms commonly used in the neuropsychology literature.

Fifteen flavonoid studies [7, 9-11, 13, 14, 21, 22, 32, 33, $35,41,48,57]$ which met the inclusion criteria were included. Ten were conducted with soy isoflavones [7, 9$11,13,21,22,32,33]$, nine of these on post-menopausal female populations [7, 9, 11, 13, 21, 22, 32, 33, 75]; two studies examined flavonoid extracts from Ginkgo biloba in older adults with [35] and without [41] AD; two studies looked at the effects of pine bark extracts on healthy older adults [48, 57]; and one study examined the effects of flavonol-rich cocoa on cognitive function in young healthy female adults [14].

Significant outcomes for cognitive testing in dietary intervention studies rely on two things: (1) the potential for cognitive change as a result of direct dietary intervention with respect to dose and duration in the cognitive domain or cognitive aspect being measured and (2) cognitive methodologies sensitive enough to measure such cognitive change. The most important consideration in setting up a suitable framework for measuring human cognitive function in flavonoid research is to determine methods that are sensitive to dietary changes and repeatable over time, simple to interpret and specific to cognitive domains. In this respect, brief measures, such as the Mini-Mental Status Examination (MMSE) [12] and the Alzheimer's Disease Assessment Scale Cognitive Subscale (ADAS-Cog) [55], 
are suitable for cognitive screening of dementia and mild cognitive impairment (MCI), a term generally used to describe the level of cognitive impairment found in the intermediate stage between normal ageing and fully developed dementia [46], and also for the measurement of widespread, gross cognitive changes over time in longitudinal studies [37]. Both tasks consist of 11-items, covering a broad range of cognitive functions: orientation, attention and calculation, memory, language and motor skills. They are less likely to be sensitive to smaller changes over shorter time periods in healthier individuals at predementia stages. In AD research, the MMSE has shown an overall progression rate of 0.24 points/month, although this was moderated by education duration, gender, disease incidence and drug therapy [54].

In the studies included in this review, a variety of approaches has been used to measure cognitive performance, targeting either multiple or single aspects of a particular cognitive domain. For instance, two isoflavone studies looked at the same four aspects of memory (verbal, visuospatial, semantic and memory span) [22, 32], whereas three others only used a single memory measure each, but combined these with measures for one or more other cognitive domains $[7,33,75]$. In contrast, others simply targeted general cognitive function as a whole (often referred to as 'global cognitive function') [35]. In total, 13 studies used multiple tasks and/or targeted multiple domains, executive function and memory, in particular [7, 9-11, 13, 21, 22, 32, 33, 41, 48, 57] (see Table 1). Of these, six studies also included at least one test of processing speed [7, 11, 22, 41, 48], and two also measured motor function $[21,75]$. In the two remaining studies [14, 35], a single task only was used. An fMRI study examined changes in BOLD signal during an executive function switching task after consumption of cocoa flavonols [14]; the AD study used a single measure of general cognition (the ADAS-Cog) to explore the effects of Gingko biloba on prevention of cognitive decline on dementia patients [35]. In this latter study, patients were screened beforehand using the MMSE. AD patients were grouped according to severity of cognitive function (e.g. MMSE score of 24 or more, $<24$ or $<15$, where lower scores indicate greater cognitive impairment). The ADAS-Cog results were analysed between cognitive impairment groups as measured by the MMSE.

\section{Results}

Across the 15 studies reported here researchers used a total of 55 separate cognitive performance measures comprising two general (or global) cognitive function tasks, three IQ measures, eight psychomotor speed tasks, 22 executive function and working memory tasks and 20 memory tasks (see Table 2). Immediate and delayed recall or recognition for the same task are counted here as a single memory task, for example, the WMS-R Logical Memory Test I and II [69].

The MMSE was used in six studies [21, 22, 32, 33, 35, 41], albeit in four as a screening tool [21, 22, 35, 41]. Three isoflavone studies [21, 33, 75] used the MMSE as a global cognitive measure, but in two cases it was non-significant [21, 32]. In the third [75], the MMSE showed a positive effect of treatment for both isoflavones and HRT versus control, which showed a negative change over time; however, the baseline mean MMSE score for the control group was $28 \pm 5.9$ (out of a possible 30 ), suggesting that individuals with MCI were included in this group which may have skewed the results. A significant effect of Ginkgo biloba treatment over 13 months was found using the ADAS-Cog [35], but only for patients with a moderate to severe cognitive impairment, such that patients with MMSE scores $<15$ showed a significantly reduced rate of cognitive decline in the treatment group compared to placebo. Nine studies measured processing speed or psychomotor speed, using eight different measures [7, 21, 22, 32, $33,41,48,57]$; two found significant treatment effects with $60 \mathrm{mg} /$ day isoflavones [7] and $180 \mathrm{mg} /$ day Ginkgo biloba [41] (see Table 1). IQ measures were used in four studies but only to check group differences at baseline [9-11, 32].

Executive function and working memory was explored in 14 studies [7, 9-11, 13, 14, 21, 22, 32, 33, 41, 48, 57] with 22 different outcome measures. Significant flavonoid treatment effects were shown in 10 of these $[7,9-11,13$, $22,33,48,57]$. In total, 10 outcome measures proved sensitive to treatment with flavonoids, although in one study, supplementation with $71 \mathrm{mg} /$ day soy milk resulted in a decline in working verbal memory performance [13]. A significant difference in an fMRI BOLD signal was found after 2 weeks supplementation with $172 \mathrm{mg} /$ day cocoa flavonols [14], but there was no corresponding behavioural effect on a switching task [14]. The CANTAB IDED test, an executive function measure of rule learning and reversal (described as assessing 'frontal function'), offered the most consistent results and was used in three isoflavone studies in young adults [11] and older adult females [9, 10], all showing significant effects of flavonoid treatment (100 and $60 \mathrm{mg} /$ day, respectively, over 612 weeks). The Stockings of Cambridge test, a measure of planning ability used in the same three studies, was significant in two of them [10, 11]. The Trail Making test, which measures 'switching' or 'shifting', was used in six studies [21, 22, 32, 33, 41], but was sensitive to flavonoidmediated cognitive change in only one isoflavone study, at a dose of $100 \mathrm{mg} /$ day [75]. Only one out of seven isoflavone studies found a significant effect of treatment on 


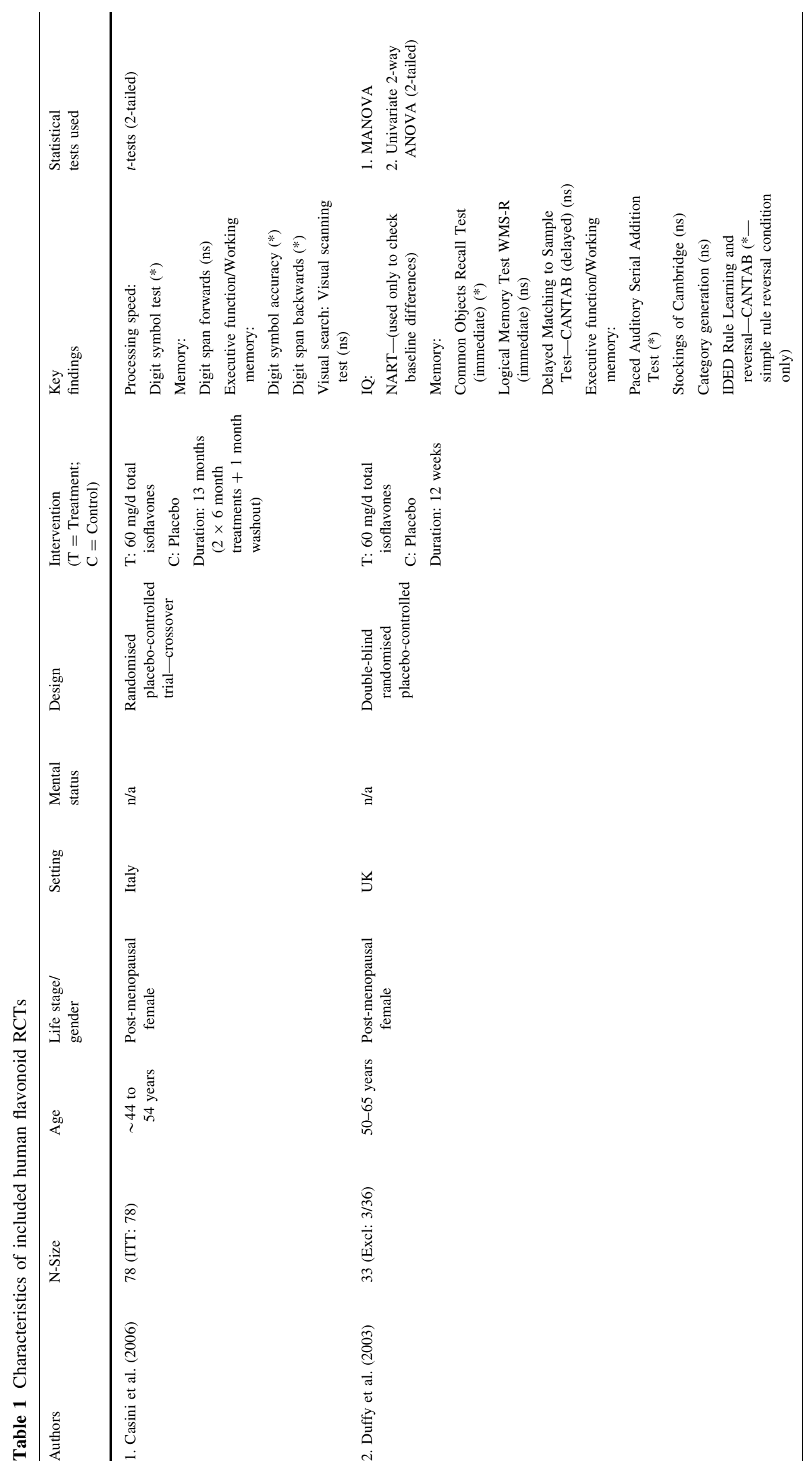




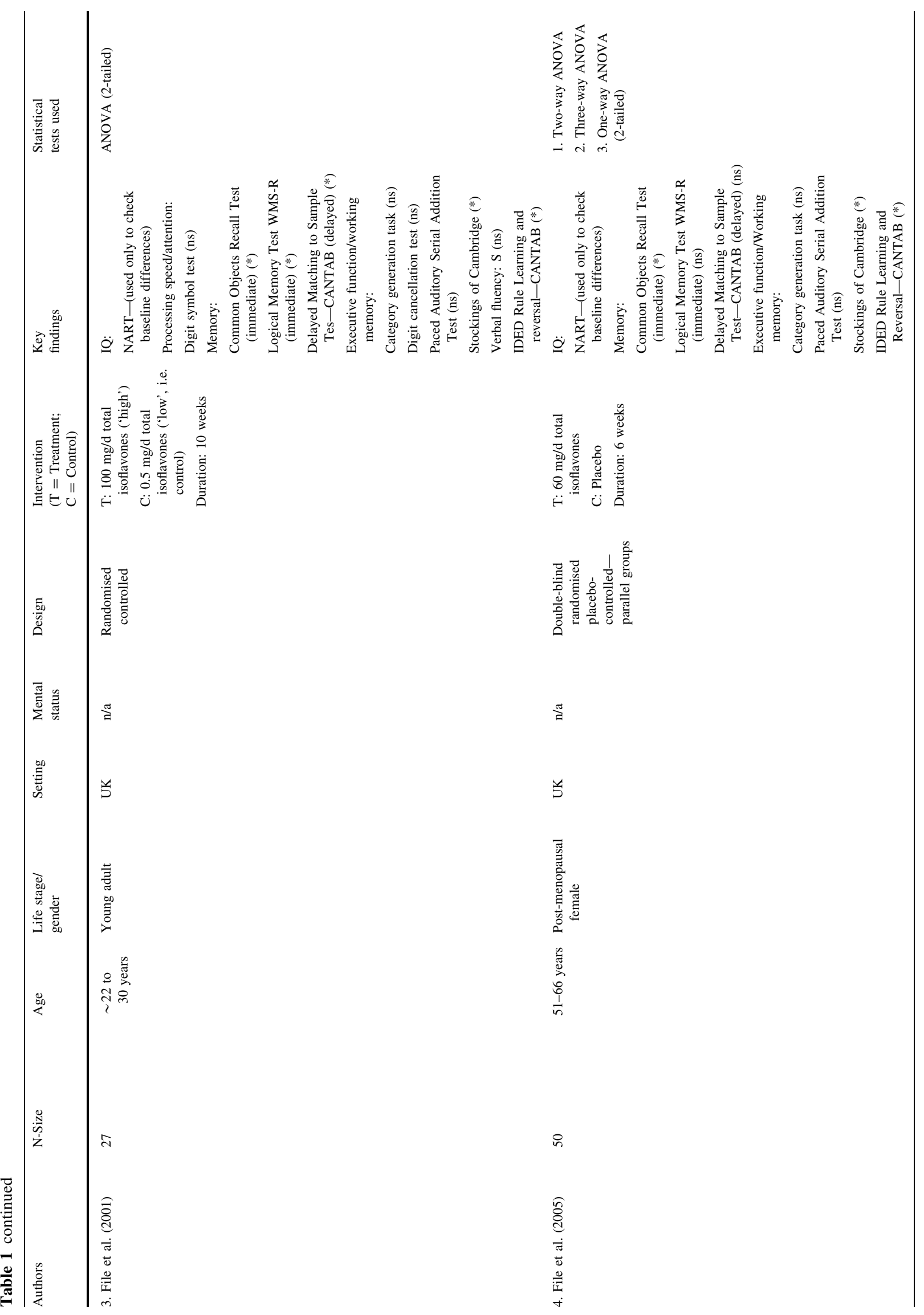




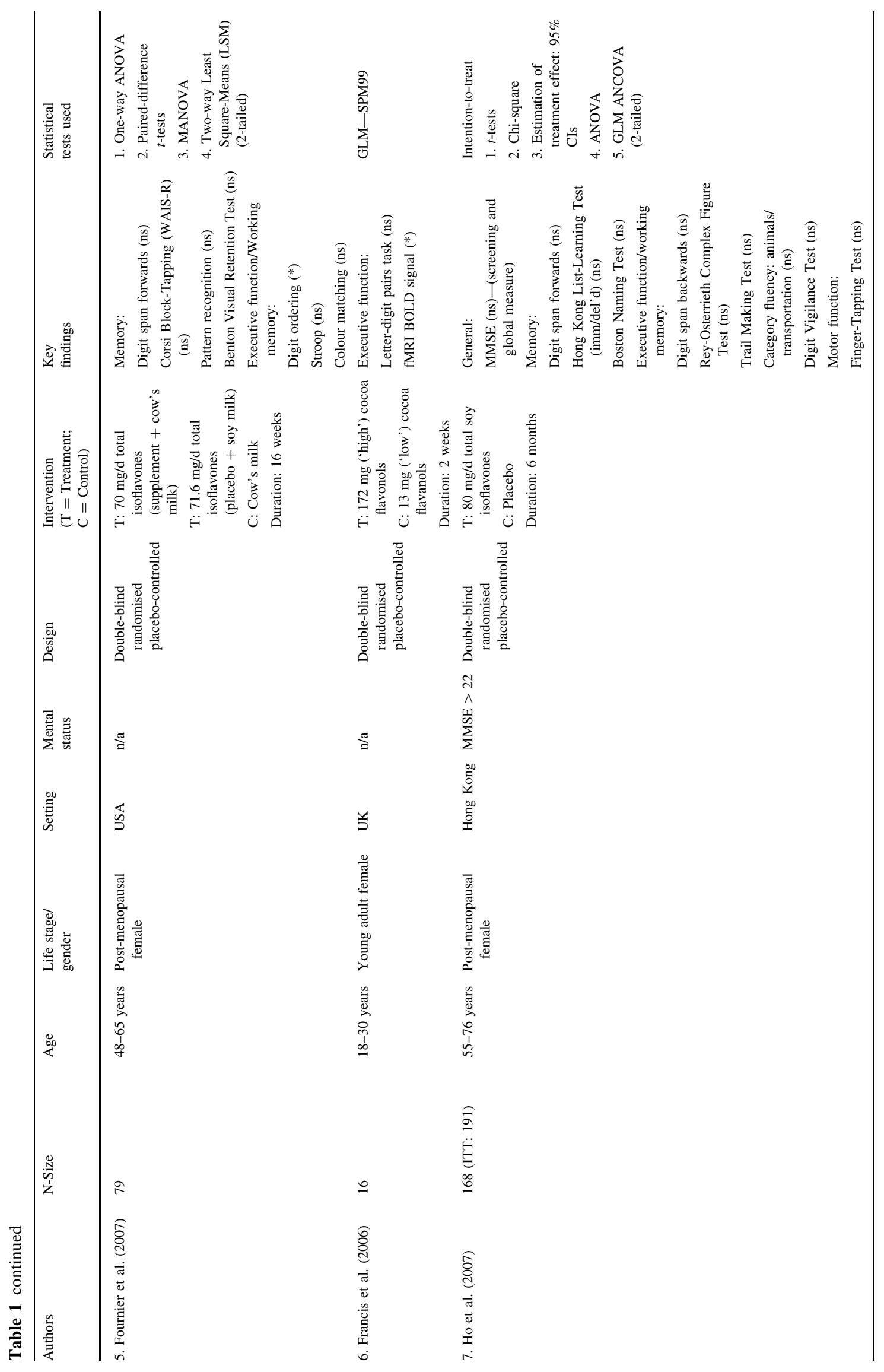




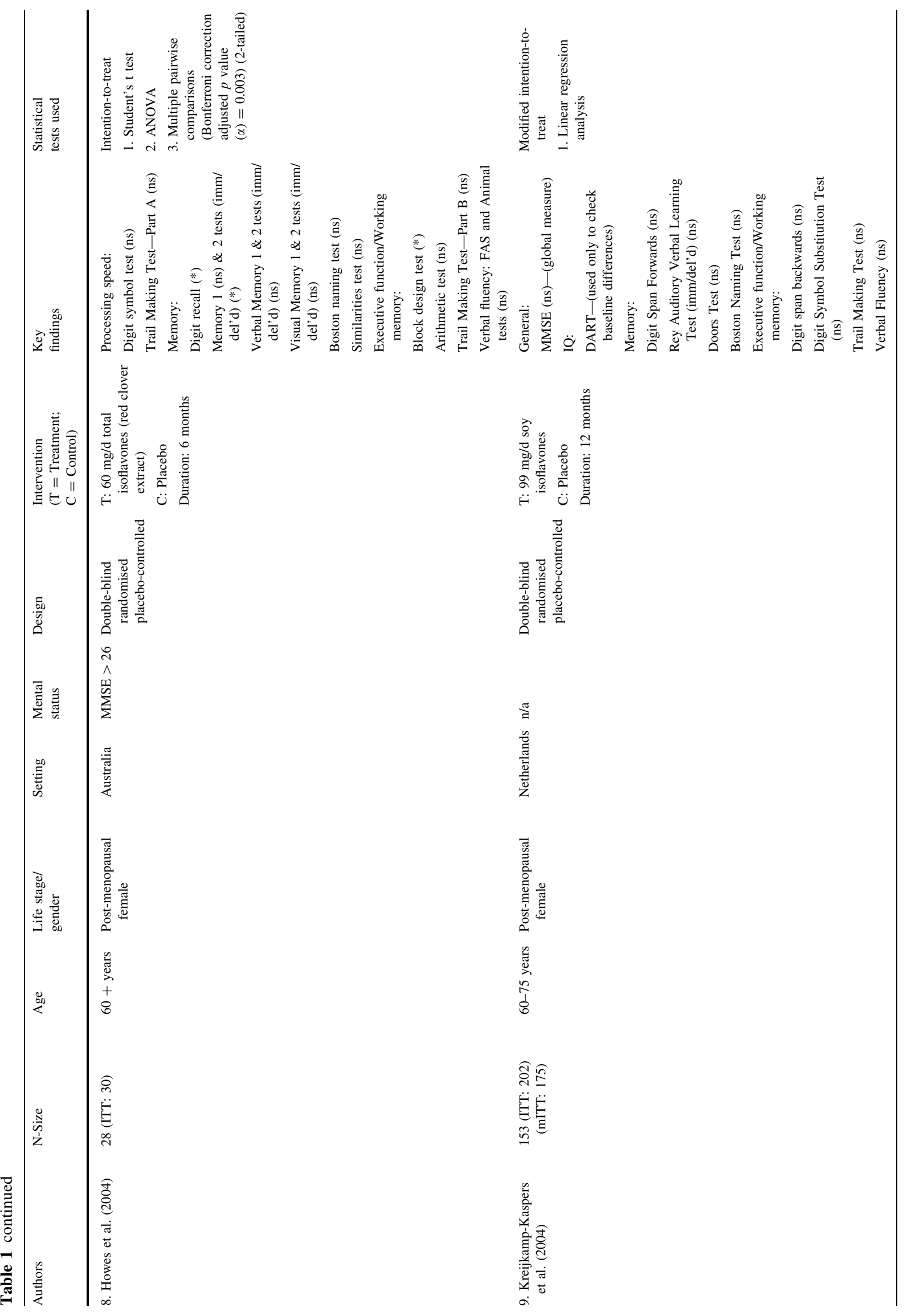




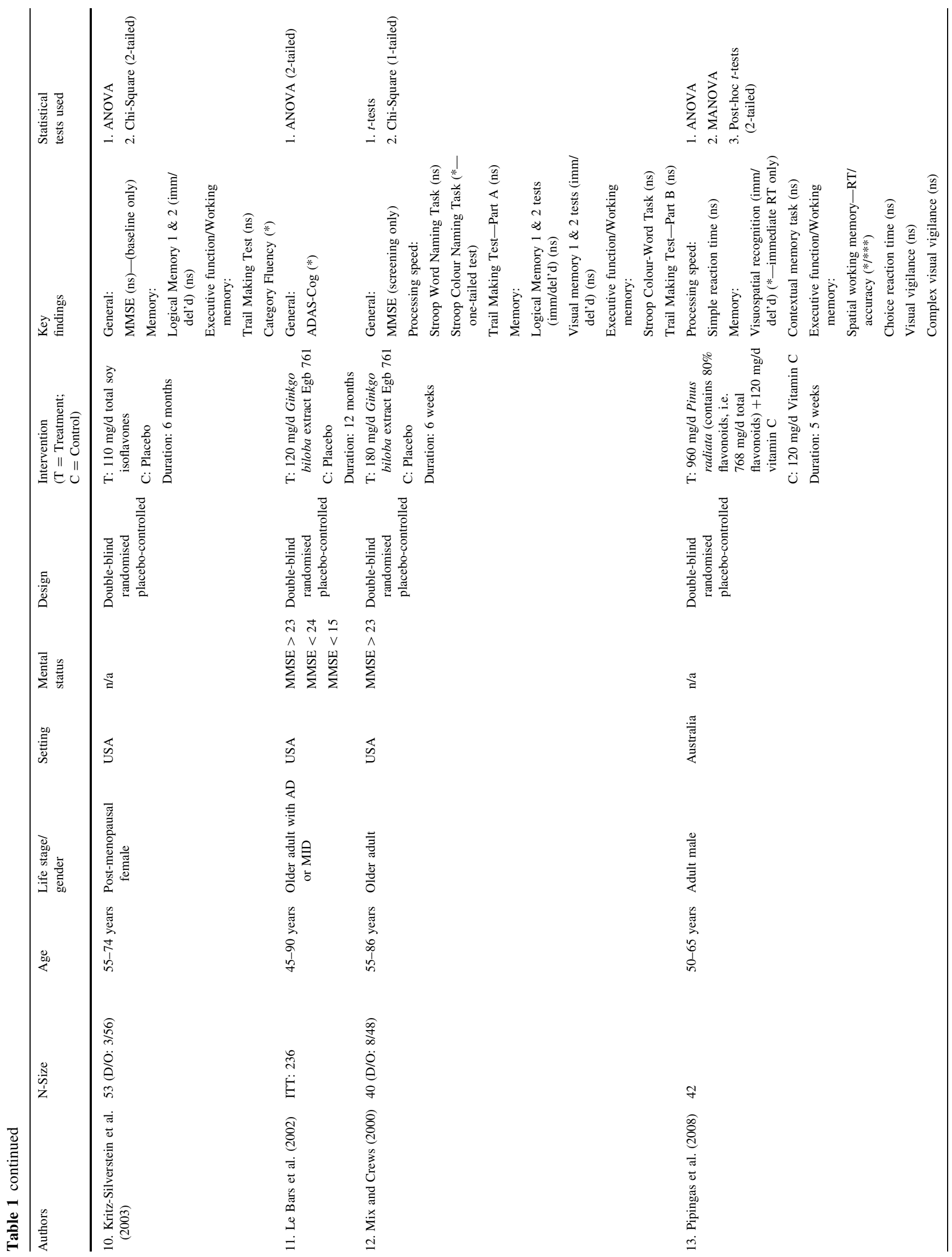




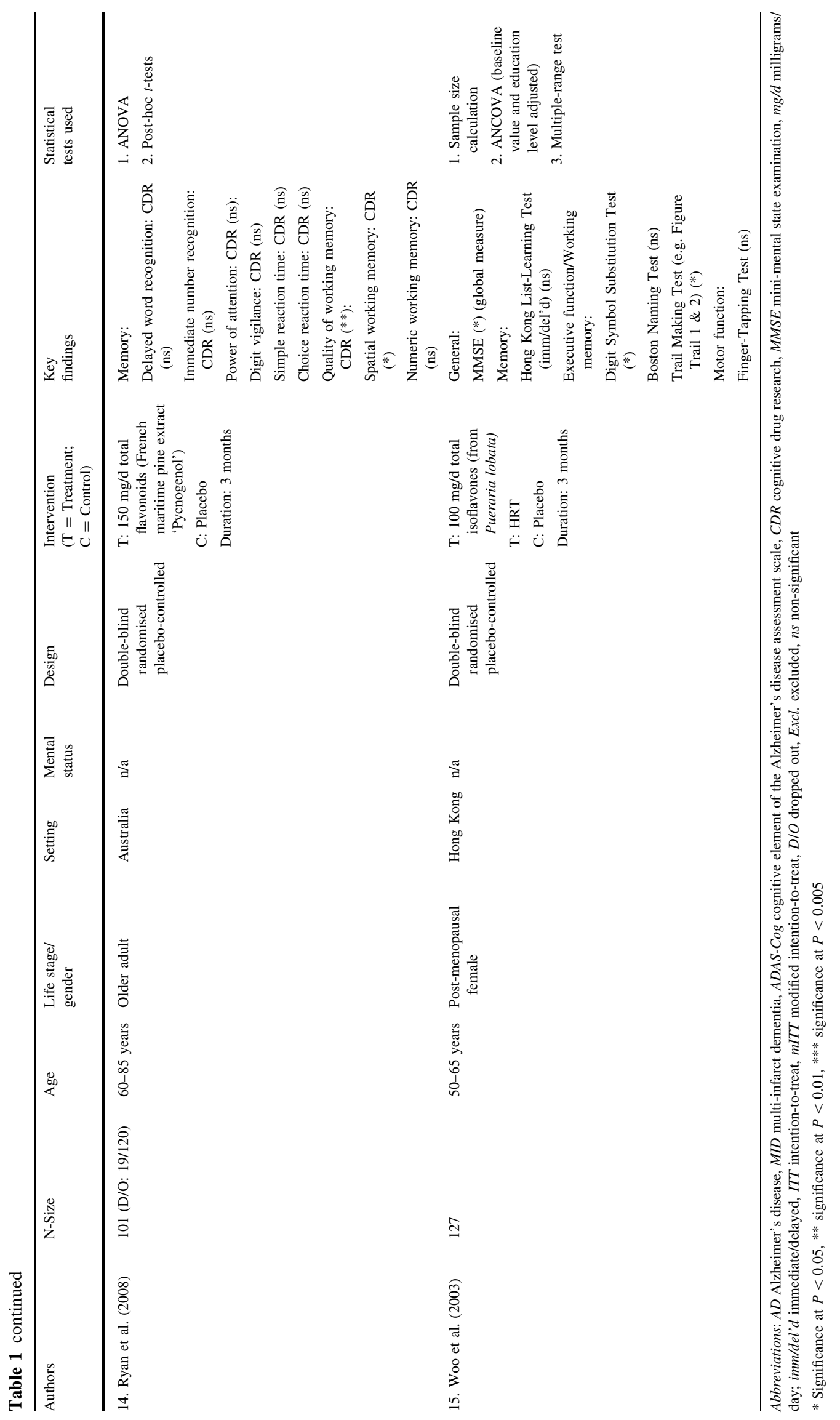


Table 2 Neuropsychological focus for 55 measures used in 14 human flavonoid RCT studies

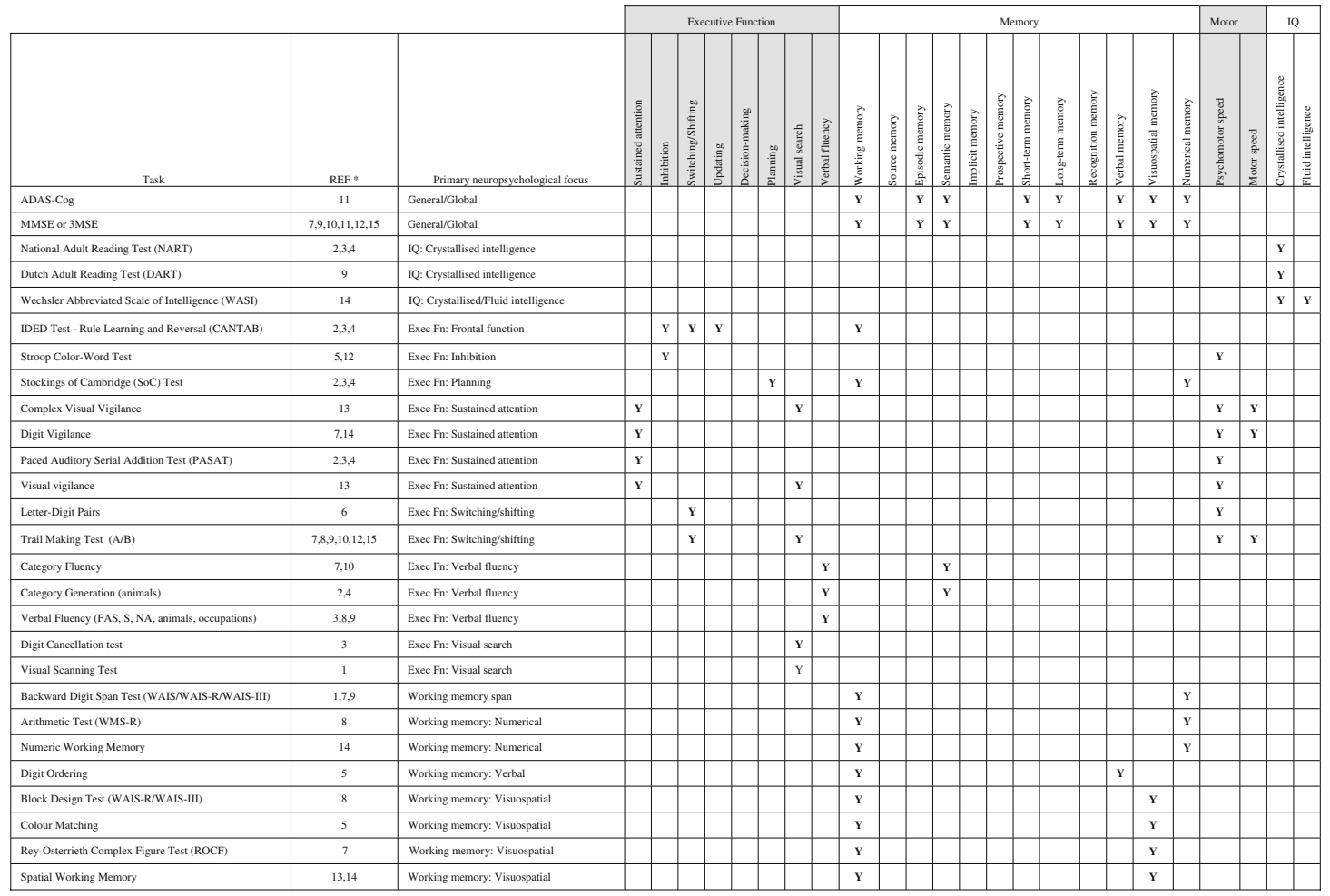

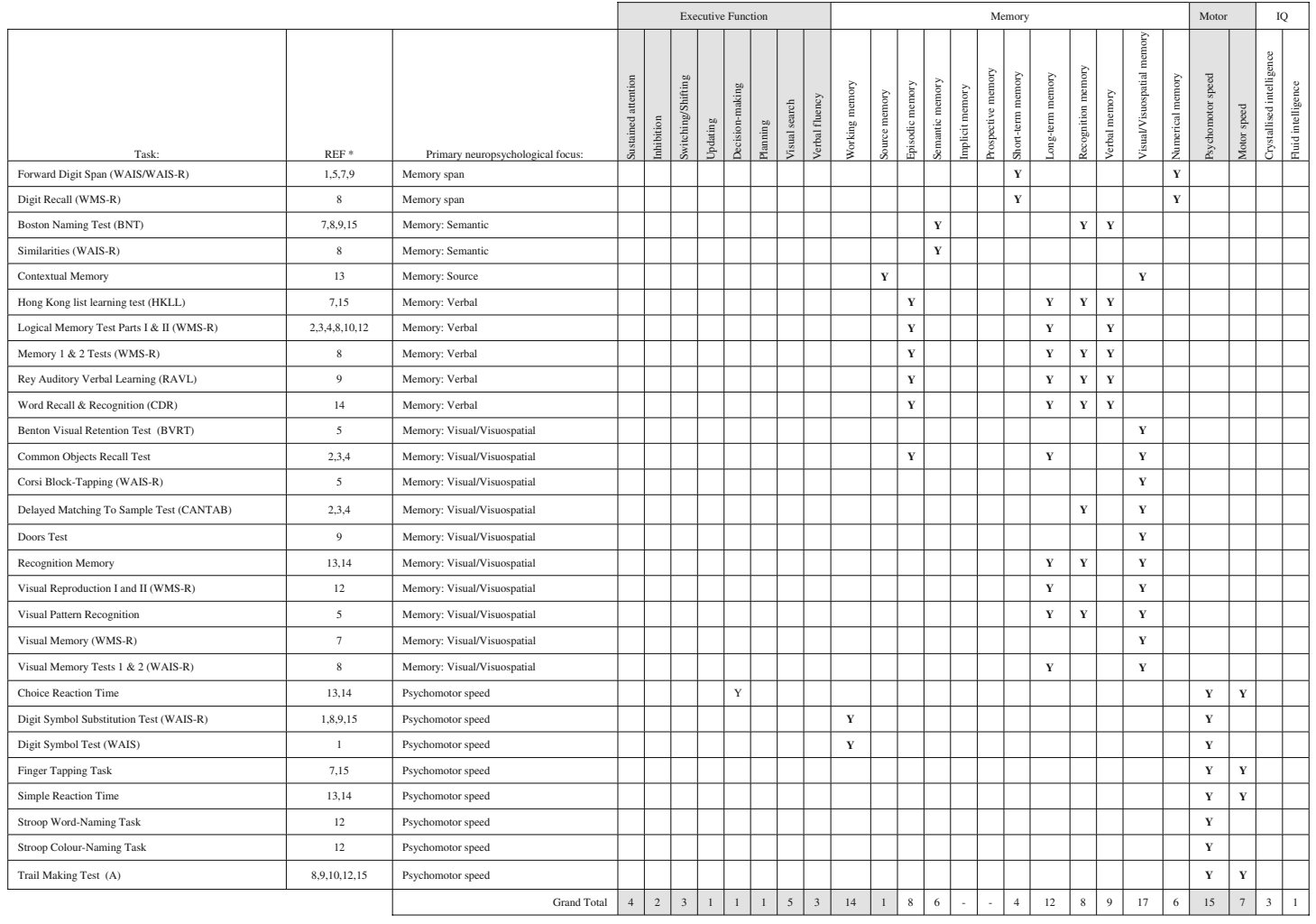

* Key to Refs.: 1 Casini et al. 2006, 2 Duffy et al. 2003, 3 File et al. 2001, 4 File et al. 2005, 5 Fournier et al. 2007, 6 Francis et al. 2006, 7 Ho et al. 2007, 8 Howes et al. 2004, 9 Kreijkamp-Kaspers et al. 2004, 10 Kritz-Silverstein et al. 2003, 11 Le Bars et al. 2002,12 Mix and Crews 2000, 13 Pipingas et al. 2008, 14 Ryan et al. 2008, 15 Woo et al. 2003 
verbal fluency using $110 \mathrm{mg} /$ day over a period of 6 months [33]. Finally, no effects of treatment were found for visual search, possibly indicating that these measures were not sensitive enough.

Working memory (numerical, verbal or visuospatial) was tested in eight studies [7, 13, 21, 22, 32, 48, 57] and showed significant effects of treatment in six of these, including the negative verbal working memory result mentioned above [7, 13, 22, 48, 57]. Spatial working memory measures accounted for three of the significant results [22, 48, 57], suggesting that findings from animal flavonoid studies are replicable in human studies.

Other memory processes were investigated using 20 different measures in 14 studies [7, 9-11, 14, 21, 22, 32, $33,35,41,48,57]$. Flavonoids were found to improve memory functioning in two isoflavone studies $[9,11]$ and in one study, using $960 \mathrm{mg} /$ day Pinus radiata [48], whereas a study using $60 \mathrm{mg} /$ day red clover extract initially found a negative effect of flavonoid treatment on long-term immediate and delayed verbal recall, and shortterm digit recall [22], which disappeared after statistical correction for error.

On closer examination of the various memory functions measured, the most consistent positive findings were shown for memory tasks with relatively short retention intervals (also described as explicit or episodic tasks, or as immediate verbal, numerical, visual or visuospatial recall). Fifteen different measures were described in 13 studies [7, 9-11, 13, 21, 22, 32, 33, 41, 48, 57, 75]. Significant positive effects of soy isoflavone treatment were found using the Common Objects recall test (a picture recall task) in young and older adults $[9,11]$, and the Logical Memory Test 1 (LM1—an immediate story recall task) in young adults [11]. Although the LM1 test was used in a number of the reviewed studies [9-11, 22, 33, 41], the above finding was not replicated elsewhere. A negative effect of $60 \mathrm{mg} /$ day isoflavone supplementation was found using the Digit Recall Test, a measure of memory span, although again this effect disappeared when the authors statistically corrected for error [22]. Longer-term memory was explored in 12 studies, using 10 different measures [ $9,13,21,22,32,33,41,48,57,75]$. The CANTAB Delayed Matching to Sample test, a measure of delayed visual recall, was the only task to reach significance in the expected direction, using $100 \mathrm{mg} /$ day isoflavone supplementation in young adults [11].

Ten RCT studies looked at visual or visuospatial memory, using 10 different measures. Only one study, using $960 \mathrm{mg} /$ day Pinus radiata showed any effect of flavonoid treatment, with improvements in reaction times on an immediate visuospatial recognition task in 50- to 65year-old males [48].

Semantic memory was investigated in four studies, but showed no significant effects of treatment [21, 22, 32, 75].
Implicit memory/learning and prospective memory were not measures in the studies reviewed here and have not to our knowledge been measured in any flavonoid RCT studies to date.

\section{Discussion}

Findings from this small selection of human cognition RCT studies using flavonoid treatments are suggestive of a positive association between flavonoid consumption and cognitive function. Although two studies had null findings and one showed a decline in cognitive outcomes, the majority of studies did find significant improvements on measures of executive function and memory, and some also found increases in general cognition and processing speed. While findings are mixed, these RCT studies clearly offer some support for previous epidemiological, longitudinal and observational findings concerning the benefits of flavonoid consumption on cognitive performance. The most interesting evidence comes from tests of specific cognitive domains, such as executive function and memory, and simple tasks focusing on specific functions within these cognitive domains have also been informative. Some of the most promising results come from studies that have used multiple measures within each cognitive domain. In contrast, general or global tests of cognitive function have been less successful when used on healthy populations. However, a great deal of work still needs to be carried out to identify tasks that are sensitive to flavonoid-related cognitive changes in healthy human populations. Interpretation of findings from existing studies is currently limited by a number of factors which we outline below.

Overall, sample sizes for the reviewed studies were relatively small, with most studies having fewer than 100 participants. Seven studies had samples of 50 or less [9-11, $14,22,41,48]$, including one which carried out 13 cognitive tasks on 28 participants (14 per treatment condition) [22], and one which carried out 10 tasks on 27 participants [11]. Obviously wherever possible sample sizes should be calculated on the basis of an expected effect size, otherwise the power of an intervention to detect a difference between treatment and placebo is at best uncertain. Power calculations and effect sizes were considered in an isoflavone study, where authors argued that 31 participants were required per group for an effect size of 0.64 and to have $70 \%$ chance to observe differences between participants [75]. Positive effects of flavonoid treatment were found for executive function, working memory and MMSE scores, although as discussed earlier, baseline measure difference may ultimately account for some of these findings. In contrast, while power calculations were discussed in another isoflavone study [22], only 15 intention-to-treat 
participants were assigned per group, and no significant results were found after correcting for error. A related issue concerns the problem of dealing with type 1 errors (e.g. the probability of rejecting the null hypothesis when the null hypothesis is true), when analysing multiple cognitive performance measures within a study. Encouragingly, three studies addressed the possibility of type 1 errors by using multiple analysis of variance (MANOVA) [9, 13, 48], and a fourth applied a Bonferroni correction when interpreting their data albeit a very conservative one [22]. A relatively large-scale soy isoflavone trial (intention-to-treat $n=175$ ) used linear regression analysis [32]. In the other studies, a series of $t$-tests [7, 41], ANOVAs, or ANCOVA [10, 11, $21,33,35,57]$ were used, with no apparent correction for multiple measures. Additionally, one of the Ginkgo biloba interventions made multiple one-tailed hypotheses, which would only be appropriate if it could be assumed that the treatment would affect all aspects of cognitive function measured; negative results published elsewhere do not support this assumption [13, 22]. In summary, researchers should be cautious when analysing and interpreting the results of multiple cognitive tests. Future studies should aim to use larger samples, carry out appropriate power calculations, consider effect sizes, and use statistical techniques that lower the risk of Type I error.

One of the aims of the current review was to try to identify particular cognitive domains and related tasks that have proven sensitive to flavonoid supplementation in existing intervention research. The major advantage of this is that it will help future researchers to select appropriate performance measures and will also begin to permit cross study comparisons of the effectiveness of different flavonoids, dose levels, populations etc. On reviewing the current studies, though, we noted that there is actually considerable confusion in the literature concerning the terms employed for the same test. As one example, the Trail Making Task Parts A and B have been referred to by different researchers as measuring visuomotor tracking and attention [33]; speed for attention, sequencing, mental flexibility, visual search and motor function [21]; information processing and prefrontal lobe function [22]; sequencing and shifting perceptual sets, concentration/ vigilance and visuomotor scanning/tracking speed [41]. While all these terms may have some validity, this inconsistency may lead to confusion for future researchers, and may hinder systematic comparison and interpretation of the specific tasks across studies.

Drawing comparisons across studies at present is clearly a challenge given the limited evidence to date and the variation in flavonoids supplemented, dose levels employed, treatment durations and delivery methods. In the isoflavone studies, doses vary between 60 and $110 \mathrm{mg} /$ day total isoflavones [7, 9-11, 13, 14, 21, 22, 32, 33]; typical isoflavone intakes reported in Asian countries are 60$100 \mathrm{mg} /$ day [13], providing a suitable rationale for the choice of dose in the majority of isoflavone studies, and findings from in vivo lipid peroxidation [74] and/or previous studies have guided others [9, 10, 21, 32]. However, other isoflavone studies do not provide explicit rationales for their choice of treatment doses. In the non-isoflavone studies, there is a wide range of flavonoid treatment doses: $172 \mathrm{mg} /$ day cocoa flavonols [14], 120 or $180 \mathrm{mg} /$ day Ginkgo biloba [35, 41], $150 \mathrm{mg} /$ day flavonoids from French maritime pine extract [57], and $768 \mathrm{mg} /$ day flavonoids from Pinus radiata in combination with $120 \mathrm{mg} /$ day vitamin C [48]. With three exceptions [21, 22, 32], baseline flavonoid levels are not reported, and cognitive change does not appear to show a relationship to dose.

Treatment durations vary from 2 weeks [14] to 13 months [7], with no obvious relationship between cognitive change and treatment duration. Most of the studies delivered flavonoids via supplements, although one trial also used soy milk [13] and another used a flavonol-rich chocolate drink [14].

As outlined earlier, previous animal studies have shown that flavonoids improve visuospatial memory (or visuospatial working memory) $[1,23,31,65,66,72]$. With one notable exception [13], there appears to be very little reference to animal flavonoid research work for clues as to 'primary dependent measures' to include in human intervention studies in this area. Of more concern, fewer than half actually investigated aspects of visuospatial memory or visuospatial working memory [13, 21, 22, 32, 48, 57]. Of the six studies that did include visuospatial tasks, three showed significant improvements for participants over the age of 50 years with either $60 \mathrm{mg} /$ day isoflavones [22], $150 \mathrm{mg} /$ day total flavonoids [57] or $960 \mathrm{mg} /$ day Pinus radiata (containing $80 \%$ flavonoids) combined with $120 \mathrm{mg} /$ day vitamin C [48].

Choice of cognitive tasks should also be guided by significant findings in previous, similar flavonoid studies, perhaps in combination with related measures targeting the particular cognitive domain of interest. Significant effects of isoflavone intake in young adults (22-30 years old) were found on tests of memory and executive function with $100 \mathrm{mg} /$ day [11]; fewer significant results were found on memory tasks using the smaller dose of $60 \mathrm{mg} /$ day isoflavones in older females [9, 10] although similar positive results were shown on the executive function tasks for all three studies [9-11]. Most of the same tests were used in these three studies; this continuity of cognitive tasks enables comparisons to be drawn across studies, and helps future studies to determine which factor will is likely to promote treatment success: isoflavone dose, age of target population or an interaction between the two. 
Most of the studies using healthy populations did not formally screen for cognitive status. Screening is an important consideration, as there is a danger of including participants with MCI within a healthy study sample, which makes interpretation difficult, as results may not be transferable to cognitively healthy populations. Of the three studies using healthy populations that did screen for cognitive impairment, all used the MMSE; one used a cut off score of 27 [22] for cognitive health, another chose 24 [41] and a third chose 23 [21]. If screening tools are to be used as standard, it is important to reach a consensus regarding cut off scores for cognitive health, as samples with lower cut off scores may include individuals with MCI. The traditional MMSE cut off score used in dementia screening is 24 , but a recent assessment of diagnostic accuracy suggests that a cut off of 27 is the optimal score for excluding cognitively impaired individuals [44].

In line with findings from animal studies which show a slowing or even a reversal of age-related decline [26, 27], the majority of the studies reviewed looked at flavonoids in the context of older populations, with the exception of the young adult isoflavone study [11] and the cocoa flavonol study with 18- to 30-year-old female students [14]. While the ongoing study of flavonoid intake in older populations is important, it is possible that the window of opportunity for slowing or reversal of age-related declines by dietary means occurs much earlier in the life-cycle, so it makes sense to also consider younger populations. Indeed, both studies showed significant differences on tasks of executive function, and the isoflavone trial also found improvements in memory tasks [11].

Finally, available evidence is limited by a lack of consideration of genetic factors which may influence shortand long-term responses to flavonoid intake, dose-response relationships, absorption, metabolism and excretion factors which influence the bioavailability and efficacy of flavonoids beneficial to cognitive function. Variations in genetic polymorphisms have been largely ignored in human flavonoid epidemiological and intervention studies. Genetic factors could explain some of the variations/inconsistencies shown in epidemiological studies, and it is important to identify polymorphisms that influence flavonoid uptake and efficacy.

\section{Conclusions}

Human flavonoid RCT research with respect to cognitive function is at a very early stage, and conclusions are not yet easy to reach. Significant benefits to cognition have been reported in the majority of studies, mainly in executive function, working memory, other memory functions and more general measures such as processing speed. However, there is little consistency across studies in terms of the cognitive domains measured, and the tasks used. While a wide range of tasks has been used, measures of important areas of everyday cognitive functioning are notable by their absence, for instance, prospective and implicit memory. Moreover, although a wide range of tasks have been used within each cognitive domain, measurement of the full range of specific functions within these domains has by no means been covered. Overall, the choice of individual outcome measures has been inconsistent, and there is general confusion as to what the various tasks actually measure. Moreover, the choice of outcome measures does not generally appear to have been guided by results from relevant animal and human flavonoid studies. There is still an over-dependence on general measures of cognitive function that have not been shown to be sensitive to shortterm changes over weeks or months in cognitively healthy populations, and which do not inform about specific cognitive functions. Some studies do not appear to have been adequately powered, as many have been carried out on small sample sizes and/or without adequately accounting for error. The wide range of treatment doses, delivery methods and intervention durations also make interpretation difficult.

These findings argue for a more systematic approach to exploring cognitive function, across multiple cognitive domains and across multiple functions within domains, addressing the omissions noted above. Overall, there is a clear and continuing need to explore more fully the relationship between flavonoid intake and cognitive function, and future studies should seek to cover the whole range of functions before a full understanding of flavonoid-mediated cognitive change can be gained. In this potentially fruitful area of human cognitive research, this can be achieved by determining cognitive outcome measures that are sensitive, simple and specific to different cognitive domains.

Acknowledgments Dr. Spencer, Dr. Butler and Dr. Williams are sponsored by the Biotechnology and Biological Sciences Research Council (BB/F008953/1). Dr. Spencer is also funded by the following BBSRC grants (BB/E023185/1; BB/G005702/1).

\section{References}

1. Andres-Lacueva C, Shukitt-Hale B, Galli RL, Jauregui O, Lamuela-Raventos RM, Joseph JA (2005) Anthocyanins in aged blueberry-fed rats are found centrally and may enhance memory. Nutr Neurosci 8:111-120

2. Baddeley A (1997) Testing executive function with a paper-andpencil test. In: Rabbitt $\mathrm{P}$ (ed) Methodology of executive and frontal lobe function. Psychology Press, Hove, pp 61-80

3. Barros D, Amaral OB, Izquierdo I, Geracitano L, Raseira M, Henriques AT, Ramirez MR (2006) Behavioral and genoprotective effects of Vaccinium berries intake in mice. Pharmacol Biochem Behav 84:229-234 
4. Burgess PW (1997) Theory and methodology in executive function research. In: Rabbitt P (ed) Methodology of frontal and executive function. Psychology Press, Hove, pp 81-116

5. Carluccio MA, Siculella L, Ancora MA, Massaro M, Scoditti E, Storelli C, Visioli F, Distante A, De Caterina R (2003) Olive oil and red wine antioxidant polyphenols inhibit endothelial activation: Antiatherogenic properties of Mediterranean diet phytochemicals. Arterioscler Thromb Vasc Biol 23:622-629

6. Casadesus G, Shukitt-Hale B, Joseph JA (2002) Qualitative versus quantitative caloric intake: are they equivalent paths to successful aging? Neurobiol Aging 23:747-769

7. Casini ML, Marelli G, Papaleo E, Ferrari A, D'Ambrosio F, Unfer V (2006) Psychological assessment of the effects of treatment with phytoestrogens on postmenopausal women: a randomized, double-blind, crossover, placebo-controlled study. Fertil Steril 85:972-978

8. Cho J, Kang JS, Long PH, Jing J, Back Y, Chung KS (2003) Antioxidant and memory enhancing effects of purple sweet potato anthocyanin and Cordyceps mushroom extract. Arch Pharm Res 26:821-825

9. Duffy R, Wiseman H, File SE (2003) Improved cognitive function in postmenopausal women after 12 weeks of consumption of a soya extract containing isoflavones. Pharmacol Biochem Behav 75:721-729

10. File SE, Hartley DE, Elsabagh S, Duffy R, Wiseman H (2005) Cognitive improvement after 6 weeks of soy supplements in postmenopausal women is limited to frontal lobe function. Menopause 12:193

11. File SE, Jarrett N, Fluck E, Duffy R, Casey K, Wiseman H (2001) Eating soya improves human memory. Psychopharmacology 157:430-436

12. Folstein MF, Folstein SE, McHugh PR (1975) Mini-mental state: practical method for grading cognitive state of patients for clinician. J Psychiatr Res 12:189-198

13. Fournier LR, Ryan BTA, Robison LM, Wiediger M, Park JS, Chew BP, McGuire MK, Sclar DA, Skaer TL, Beerman KA (2007) The effects of soy milk and isoflavone supplements on cognitive performance in healthy, postmenopausal women. J Nutr Health Aging 11:155

14. Francis ST, Head K, Morris PG, Macdonald IA (2006) The effect of flavanol-rich cocoa on the fMRI response to a cognitive task in healthy young people. J Cardiovasc Pharmacol 47:S215-S220

15. Frick M, Weidinger F (2007) Endothelial function: a surrogate endpoint in cardiovascular studies? Curr Pharm Design 13:17411750

16. Galli RL, Shukitt-Hale B, Youdim KA, Joseph JA (2002) Fruit polyphenolics and brain aging: nutritional interventions targeting age-related neuronal and behavioral deficits. Ann NY Acad Sci 959:128-132

17. Gonzalez-Gallego J, Sanchez-Campos S, Tunon MJ (2007) Antiinflammatory properties of dietary flavonoids. Nutr Hosp 22:287293

18. Goyarzu F, Malin DH, Lau FC, Taglialatela G, Moon WD, Jennings R, Moy E, Moy D, Lippold S, Shukitt-Hale B, Joseph JA (2004) Blueberry supplemented diet: effects on object recognition memory and nuclear factor-kappa B levels in aged rats. Nutr Neurosci 7:75-83

19. Harborne JB, Williams CA (2000) Advances in flavonoid research since 1992. Phytochemistry 55:481-504

20. Hishikawa K, Nakaki T, Fujita T (2005) Oral flavonoid supplementation attenuates atherosclerosis development in apolipoprotein E-deficient mice. Arterioscler Thromb Vasc Biol 25:442-446

21. Ho SC, Chan ASY, Ho YP, So EKF, Sham A, Zee B, Woo JLF (2007) Effects of soy isoflavone supplementation on cognitive function in Chinese postmenopausal women: a double-blind, randomized, controlled trial. Menopause 14:489
22. Howes JB, Bray K, Lorenz L, Smerdely P, Howes LG (2004) The effects of dietary supplementation with isoflavones from red clover on cognitive function in postmenopausal women. Climacteric 7:70-77

23. Huang JL, Fu ST, Jiang YY, Cao YB, Guo ML, Wang Y, Xu Z (2007) Protective effects of Nicotiflorin on reducing memory dysfunction, energy metabolism failure and oxidative stress in multi-infarct dementia model rats. Pharmacol Biochem Behav 86:741-748

24. Joseph JA, Shukitt-Hale B, Lau FC (2007) Fruit polyphenols and their effects on neuronal signaling and behavior in senescence. Ann NY Acad Sci 1100:470-485

25. Joseph JA, Denisova NA, Bielinski D, Fisher DR, Shukitt-Hale B (2000) Oxidative stress protection and vulnerability in aging: putative nutritional implications for intervention. Mech Ageing Dev 116:141-153

26. Joseph JA, Denisova N, Fisher D, Shukitt-Hale B, Bickford P, Prior R, Cao GH (1998) Age-related neurodegeneration and oxidative stress: putative nutritional intervention. Neurol Clin 16:747-755

27. Joseph JA, Shukitt-Hale B, Denisova NA, Bielinski D, Martin A, McEwen JJ, Bickford PC (1999) Reversals of age-related declines in neuronal signal transduction, cognitive, and motor behavioral deficits with blueberry, spinach, or strawberry dietary supplementation. J Neurosci 19:8114-8121

28. Joseph JA, Shukitt-Hale B, Denisova NA, Prior RL, Cao G, Martin A, Taglialatela G, Bickford PC (1998) Long-term dietary strawberry, spinach, or vitamin E supplementation retards the onset of age-related neuronal signal-transduction and cognitive behavioral deficits. J Neurosci 18:8047-8055

29. Kim DH, Jeon SJ, Son KH, Jung JW, Lee S, Yoon BH, Choi JW, Cheong JH, Ko KH, Ryu JH (2006) Effect of the flavonoid, oroxylin $\mathrm{A}$, on transient cerebral hypoperfusion-induced memory impairment in mice. Pharmacol Biochem Behav 85:658-668

30. Knapp M, Prince M, Albanese E, Banerjee S, Dhanasiri S, Fernandez JL, Ferri C, McCrone P, Snell T, Stewart R (2007) Dementia UK: a report into the prevalence and cost of dementia prepared by the Personal social Services Research Unit (PCSSRU) at the London School of Economics and Institute of Psychiatry at King's College London for the Alzheimer's Society. Alzheimer's Society, London

31. Koda T, Kuroda Y, Imai H (2008) Protective effect of rutin against spatial memory impairment induced by trimethyltin in rats. Nutr Res 28:629-634

32. Kreijkamp-Kaspers S, Kok L, Grobbee DE, de Haan EHF, Aleman A, Lampe JW, van der Schouw YT (2004) Effect of soy protein containing isoflavones on cognitive function, bone mineral density, and plasma lipids in postmenopausal women: a randomized controlled trial. J Am Med Assoc 292:65-74

33. Kritz-Silverstein D, Von Muhlen D, Barrett-Connor E, Bressel MAB (2003) Isoflavones and cognitive function in older women: the SOy and Postmenopausal Health in Aging (SOPHIA) Study. Menopause 10:196

34. Lau FC, Shukitt-Hale B, Joseph JA (2005) The beneficial effects of fruit polyphenols on brain aging. Neurobiol Aging 26:S128S132

35. Le Bars PL, Velasco FM, Ferguson JM, Dessain EC, Kieser M, Hoerr R (2002) Influence of the severity of cognitive impairment on the effect of the Ginkgo biloba extract EGb $761^{\circledR}$ in Alzheimer's disease. Neuropsychobiology 45:19-26

36. Lee YB, Lee HJ, Sohn HS (2005) Soy isoflavones and cognitive function. J Nutr Biochem 16:641-649

37. Letenneur L, Proust-Lima C, Le Gouge A, Dartigues JF, Barberger-Gateau P (2007) Flavonoid intake and cognitive decline over a 10-year period. Am J Epidemiol 165:1364-1371 
38. Manach C, Scalbert A, Morand C, Remesy C, Jimenez L (2004) Polyphenols: food sources and bioavailability. Am J Clin Nutr 79:727-747

39. Mangels AR, Messina V, Melina V (2003) Position of the American Dietetic Association and Dieticians of Canada: vegetarian diets. J Am Diet Assoc 103:748-765

40. Manly T, Robinson IH (1997) Sustained attention and the frontal lobes. In: Rabbitt P (ed) Methodology of executive and frontal lobe function. Psychology Press, Hove, pp 135-154

41. Mix JA, Crews WD Jr (2000) An examination of the efficacy of Ginkgo biloba extract EGb 761 on the neuropsychologic functioning of cognitively intact older adults. J Altern Complement Med 6:219-229

42. Miyake A, Friedman NP, Emerson MJ, Witzki AH, Howerter A, Wager TD (2000) The unity and diversity of executive functions and their contributions to complex "frontal lobe" tasks: a latent variable analysis. Cogn Psychol 41:49-100

43. Moser DJ, Hoth KF, Robinson RG, Paulsen JS, Sinkey CA, Benjamin ML, Schultz SK, Haynes WG (2004) Blood vessel function and cognition in elderly patients with atherosclerosis. Stroke 35:E369-E372

44. O'Bryant SE, Humphreys JD, Smith GE, Ivnik RJ, Graff-Radford NR, Petersen RC, Lucas JA (2008) Detecting dementia with the mini-mental state examination in highly educated individuals. Arch Neurol 65:963

45. Passamonti S, Vrhovsek U, Vanzo A, Mattivi F (2005) Fast access of some grape pigments to the brain. J Agric Food Chem 53:7029-7034

46. Petersen RC, Smith GE, Waring SC, Ivnik RJ, Tangalos EG, Kokmen E (1999) Mild cognitive impairment clinical characterization and outcome. Am Med Assoc 56:303-308

47. Phillips LH (1997) Do "frontal tests" measure executive function? Issues of assessment and evidence from fluency tests. In: Rabbitt P (ed) Methodology of frontal and executive function. Psychology Press, Hove, pp 191-213

48. Pipingas A, Silberstein RB, Vitetta L, Van Rooy C, Harris EV, Young JM, Frampton CM, Sali A, Nastasi J (2008) Improved cognitive performance after dietary supplementation with a pinus radiata bark extract formulation. Phytother Res 22:1168-1174

49. Rabbitt P (1997) Methodology of frontal and executive function. Psychology Press, Hove

50. Rai GS, Shovlin C, Wesnes KA (1991) A double-blind, placebo controlled-study of Ginkgo biloba extract (tanakan) in elderly outpatients with mild to moderate memory impairment. Curr Med Res Opin 12:350-355

51. Ramirez MR, Izquierdo I, Raseira MDB, Zuanazzi JA, Barros D, Henriques AT (2005) Effect of lyophilised Vaccinium berries on memory, anxiety and locomotion in adult rats. Pharmacol Res $52: 457-462$

52. Reed J (2002) Cranberry flavonoids, atherosclerosis and cardiovascular health. Crit Rev Food Sci Nutr 42:301-316

53. Rohdewald P (2002) A review of the French maritime pine bark extract $\left(\right.$ Pycnogenol ${ }^{\circledR}$ ), a herbal medication with a diverse clinical pharmacology. Int J Clin Pharmacol Ther 40:158-168

54. Roselli F, Tartaglione B, Federico F, Lepore V, Defazio G, Livrea P (2009) Rate of MMSE score change in Alzheimer's disease: influence of education and vascular risk factors. Clin Neurol Neurosurg 111:327-330

55. Rosen WG, Mohs RC, Davis KL (1984) A new rating-scale for Alzheimer's-Disease. Am J Psychiatry 141:1356-1364

56. Rugg MD, Henson RNA (2002) Episodic memory retrieval: an (event-related) functional neuroimaging perspective. In: Parker AE, Wilding EL, Bussey T (eds) The cognitive neuroscience of memory encoding and retrieval. Psychology Press, Hove, pp 83-99

57. Ryan J, Croft K, Mori T, Wesnes K, Spong J, Downey L, Kure C, Lloyd J, Stough C (2008) An examination of the effects of the antioxidant Pycnogenol ${ }^{\circledR}$ on cognitive performance, serum lipid profile, endocrinological and oxidative stress biomarkers in an elderly population. J Psychopharmacol 22:553

58. Sattar N, Ferns G (2005) Endothelial dysfunction. In: Stanner S (ed) Cardiovascular disease: diet, nutrition and emerging risk factors. Blackwell Publishing, Oxford, pp 63-77

59. Shang YZ, Qin BW, Cheng JJ, Miao H (2006) Prevention of oxidative injury by flavonoids from stems and leaves of Scutellaria baicalensis Georgi in PC12 cells. Phytother Res 20:53-57

60. Shukitt-Hale B, Carey A, Simon L, Mark DA, Joseph JA (2006) Effects of Concord grape juice on cognitive and motor deficits in aging. Nutrition 22:295-302

61. Spencer JP (2007) The interactions of flavonoids within neuronal signalling pathways. Genes Nutr 2:257-273

62. Spencer, JPE (2008) Flavonoids: modulators of brain function? Br J Nutr 99(E-Suppl 1):ES60-ES77

63. Spencer JPE, Whiteman M, Jenner P, Halliwell B (2002) 5-S-cysteinyl-conjugates of catecholamines induce cell damage, extensive DNA base modification and increases in caspase- 3 activity in neurons. J Neurochem 81:122-129

64. Spencer JPE, Jenner P, Daniel SE, Lees AJ, Marsden DC, Halliwell B (1998) Conjugates of catecholamines with cysteine and GSH in Parkinson's disease: possible mechanisms of formation involving reactive oxygen species. J Neurochem $71: 2112-2122$

65. Stackman RW, Eckenstein F, Frei B, Kulhanek D, Nowlin J, Quinn JF (2003) Prevention of age-related spatial memory deficits in a transgenic mouse model of Alzheimer's disease by chronic Ginkgo biloba treatment. Exp Neurol 184:510-520

66. Sun SW, Yu HQ, Zhang H, Zheng YL, Wang JJ, Luo L (2007) Quercetin attenuates spontaneous behavior and spatial memory impairment in d-galactose-treated mice by increasing brain antioxidant capacity. Nutr Res 27:169-175

67. Tagawa H, Tomoike H, Nakamura M (1991) Putative mechanisms of the impairment of endothelium-dependent relaxation of the aorta with atheromatous plaque in heritable hyperlipidemic rabbits. Circ Res 68:330-337

68. Vauzour D, Vafeiadou K, Rodriguez-Mateos A, Rendeiro C, Spencer JPE (2008) The neuroprotective potential of flavonoids: a multiplicity of effects. Genes Nutr 3:115-126

69. Wechsler D (1981) WAIS-R manual: Wechsler adult intelligence scale-revised. The Psychological Corporation, New York

70. Weinreb O, Mandel S, Amit T, Youdim MBH (2004) Neurological mechanisms of green tea polyphenols in Alzheimer's and Parkinson's diseases. J Nutr Biochem 15:506-516

71. WHO (2007) Medium-term strategic plan 2008-2013 and proposed programme budget 2008-2009. World Health Organization, Geneva

72. Williams CM, El Mohsen MA, Vauzour D, Rendeiro C, Butler LT, Ellis JA, Whiteman M, Spencer JPE (2008) Blueberryinduced changes in spatial working memory correlate with changes in hippocampal CREB phosphorylation and brainderived neurotrophic factor (BDNF) levels. Free Radic Biol Med 45:295-305

73. Williams RJ, Spencer JPE, Rice-Evans C (2004) Flavonoids: antioxidants or signalling molecules? Free Radic Biol Med 36:838-849

74. Wiseman H, JD OReilly, Adlercreutz H, Mallet A, Bowey EA, Rowland IR, Sanders TA (2000) Isoflavone phytoestrogens consumed in soy decrease F 2-isoprostane concentrations and increase resistance of low-density lipoprotein to oxidation in humans. Am J Clin Nutr 72:395-400

75. Woo J, Lau E, Ho SC, Cheng F, Chan C, Chan ASY, Haines CJ, Chan TYK, Li MMP, Sham A (2003) Comparison of Pueraria lobata with hormone replacement therapy in treating the adverse health consequences of menopause. Menopause 10:352 